\title{
Factores que afectan el posicionamiento de productos en el exterior: el caso del sector floricultor antioqueño ${ }^{1}$
}

Factors affecting the positioning of products abroad: the case of the Antioquian floriculture sector

Recibido: 12 de mayo de 2015 - Revisado: 20 de octubre de 2015 - Aceptado: 10 de noviembre de 2015

Juan Gabriel Vanegas ${ }^{2}$

Jorge A. Restrepo ${ }^{3}$

\section{Resumen}

El desempeño exportador de una firma depende de factores internos y externos. Este artículo presenta un ejercicio de valoración de factores que inciden en las exportaciones del sector floricultor del departamento de Antioquia, Colombia. Para ello, se sigue un método de decisiones jerárquico apoyado en juicios de expertos con el concurso de empresarios, gremios e instituciones. Los resultados indican que la visión empresarial limitada, el entrenamiento del capital humano, la capacidad interna logística de la organización y la volatilidad del tipo de cambio constituyen los principales limitantes que encara el sector para su internacionalización vía exportaciones. Los hallazgos de la investigación sirven de propuesta para validar una metodología que permita priorizar los factores que condicionan las decisiones de expansión internacional.

\section{Palabras clave}

Barreras a la exportación, sector floricultor, proceso análisis jerárquico, Antioquia.

\section{Abstract}

The export performance of a firm depends on internal and external factors. This article presents a valuation exercise of factors affecting exports of the floriculture sector of the department of Antioquia, Colombia. To this end, we have followed a method of hierarchical decisions supported by expert judgments with the help of employers, unions and institutions. The results indicate that the limited business vision, the human capital training, the internal logistics capacity of the organization and the exchange rate volatility are the main constraints the sector faces for internationalization through exports. The research findings serve as a proposal to validate a methodology that allows to prioritize the factors that influence the decisions of international expansion.

\section{Keywords}

Export barriers, floriculture sector, hierarchical analysis process, Antioquia.
1 Este artículo es producto del proyecto de investigación: "Perspectivas de la toma de decisiones en agentes económicos: aplicaciones en contextos de negocios y turismo"; proyecto conjunto desarrollado entre el Tecnológico de Antioquia Institución Universitaria, Medellín, Colombia; y la Fundación Universitaria Autónoma de las Américas, Medellín, Colombia. Los autores agradecen la participación en el proceso de recolección de información de Hans Rossemberth Gómez Burbano y Roberto Antonio Ocampo Vargas.

2 Economista. MSc. en Economía. Docente-investigador en el Tecnológico de Antioquia Institución Universitaria. Facultad de Ciencias Administrativas y Económicas, Grupo de Investigación RED, Medellín, Colombia.

Correo electrónico: jvanegas1@tdea.edu.co, jg.tecnologico@gmail.com.

${ }_{3}^{3}$ Ph.D. MBA. Ingeniero administrador. Docente-investigador en la Fundación Universitaria Autónoma de las Américas. Facultad de Ciencias Económicas y Administrativas, Grupo de Investigación Gicea, Medellín, Colombia.

Correo electrónico:

jorge.restrepo@uam.edu.co, gifatdea@gmail.com

Para citar este artículo use: Vanegas, J., \& Restrepo, J. (2016). Factores que afectan el posicionamiento de productos en el exterior: el caso del sector floricultor antioqueño. Revista Civilizar Ciencias Sociales y Humanas, 16(30), 145-160. 


\section{Introducción}

La floricultura colombiana ha mostrado durante los últimos cuatro decenios un comportamiento dinámico logrando posicionamiento en los mercados internacionales. Los resultados ubican el sector como el segundo proveedor mundial de flores frescas, el primero en claveles y el más importante abastecedor del mercado norteamericano (Asocolfores, 2009). En el plano macroeconómico, las fluctuaciones cambiarias han actuado como un factor determinante de la competitividad del sector; al igual que la creciente competencia mundial, las variaciones relativas de precios y restricciones en la demanda, que se suman a los problemas que inciden en el desempeño del ramo (Calderón, 2014; Reina \& Acosta, 2008).

El sector floricultor colombiano encontró en la década de los setenta una oportunidad excelente de inversión y un futuro alentador, como consecuencia de las ventajas internas y externas para iniciar en la agricultura comercial moderna y continuar como fuente generadora de empleo directo en el campo (Quirós, 2001). En la actualidad este sector se ubica en un puesto privilegiado en el ámbito internacional porque desde sus orígenes, por la calidad de sus productos y las ventajas comparativas, se pensó como exportador $\mathrm{y}$ ha sorteado mercados exigentes que llevaron a los floricultores a innovar en los sistemas de cultivo, cosecha y poscosecha al unísono con los estándares de los países compradores como Estados Unidos, Reino Unido, Canadá y Japón, entre otros (González, 2013).

En ese sentido, la actividad de exportar es quizá la forma más popular, rápida y sencilla de internacionalización para una compañía, en particular, de tamaño pequeño (Cavusgil \& Kirpalani, 1993; Zafarullah, Mujahid, \& Young, 1998). No obstante, la presencia de ciertos elementos puede obstaculizar el desarrollo de esta ocupación. Las revisiones sistemáticas a los estudios empíricos de barreras a la exportación las han clasificado tanto desde los aspectos internos que competen al margen de maniobra de una organización, hasta aquellos factores propios del entorno (Doern, 2009; Kaleka \& Katsikeas, 1995; Katsikeas \& Morgan, 1994; Leonidou, 1995a; Tesfom \& Lutz, 2006). También se plantea la existencia de obstáculos de tipo actitudinal, estructural, operativo y de otra índole, que se circunscriben en el concepto de barreras a la exportación, y que influyen en la decisión de una firma de empezar, desarrollar o permanecer en el campo del comercio internacional (Leonidou, 1995a).

Otra categoría de análisis, expone cómo las fluctuaciones cambiarias son determinantes en la competitividad de empresas, sectores y países, así como la dinámica ascendente de la competencia mundial, las variaciones relativas de precios y las restricciones en la demanda, se clasifican como barreras que inciden en su desempeño exportador. Con frecuencia, dichas barreras direccionan el fracaso de las empresas en sus estrategias de posicionamiento más allá de las fronteras nacionales, minando su estructura financiera y acumulando externalidades con impactos negativos sobre las actividades presentes y futuras de los exportadores (Leonidou, 1995a).

En consecuencia, este trabajo presenta un ejercicio de valoración de factores y barreras que afectan y catalizan el proceso exportador del sector floricultor en el departamento de Antioquia (Colombia). Para ello, esta investigación aplica una metodología mixta, donde se determinó la percepción de expertos en torno a las barreras que obstaculizan el desarrollo de las empresas floricultoras en Antioquia. Simultáneamente, se utiliza la técnica de análisis jerárquico de procesos, para categorizar los obstáculos y establecer una escala en orden de importancia relativa, y así determinar el nivel de impacto de estos sobre los principales destinos de exportación. Para la recolección de información se procedió con entrevistas estructuradas a una muestra no aleatoria intencionada de seis expertos. 
Este estudio se organiza en seis secciones incluida la introducción. La segunda revela un panorama general de los resultados del sector floricultor. La tercera sección muestra los acercamientos empíricos al problema de las barreras a la exportación. En cuarto lugar se detalla la metodología que se siguió. Los resultados y su discusión son abordados en la sección quinta. Al final se esbozan las conclusiones.

\section{Contexto del sector floricultor}

El desempeño exportador del sector floricultor antioqueño en cuanto a la participación de este dentro de las exportaciones totales ha fluctuado entre el $2,1 \%$ y el $4,3 \%$, para un valor promedio anual de $3,7 \%$. Los flujos de productos de plantas vivas y productos de floricultura en el departamento se han expandido rápidamente en los últimos años, en particular después de la segunda mitad de la primera década del 2000, y en el último año ha tocado su techo máximo tanto en valor como en volumen; cifras que en valor se han multiplicado por un poco más de 4,5 veces desde 1998 (véase gráfica 1). La tasa de crecimiento promedio anual del sector ha sido de 10,9 , ligeramente por debajo del registrado por las exportaciones totales, $14,2 \%$.

Gráfica 1

Comportamiento de las exportaciones de flores en Antioquia, 1998-2014

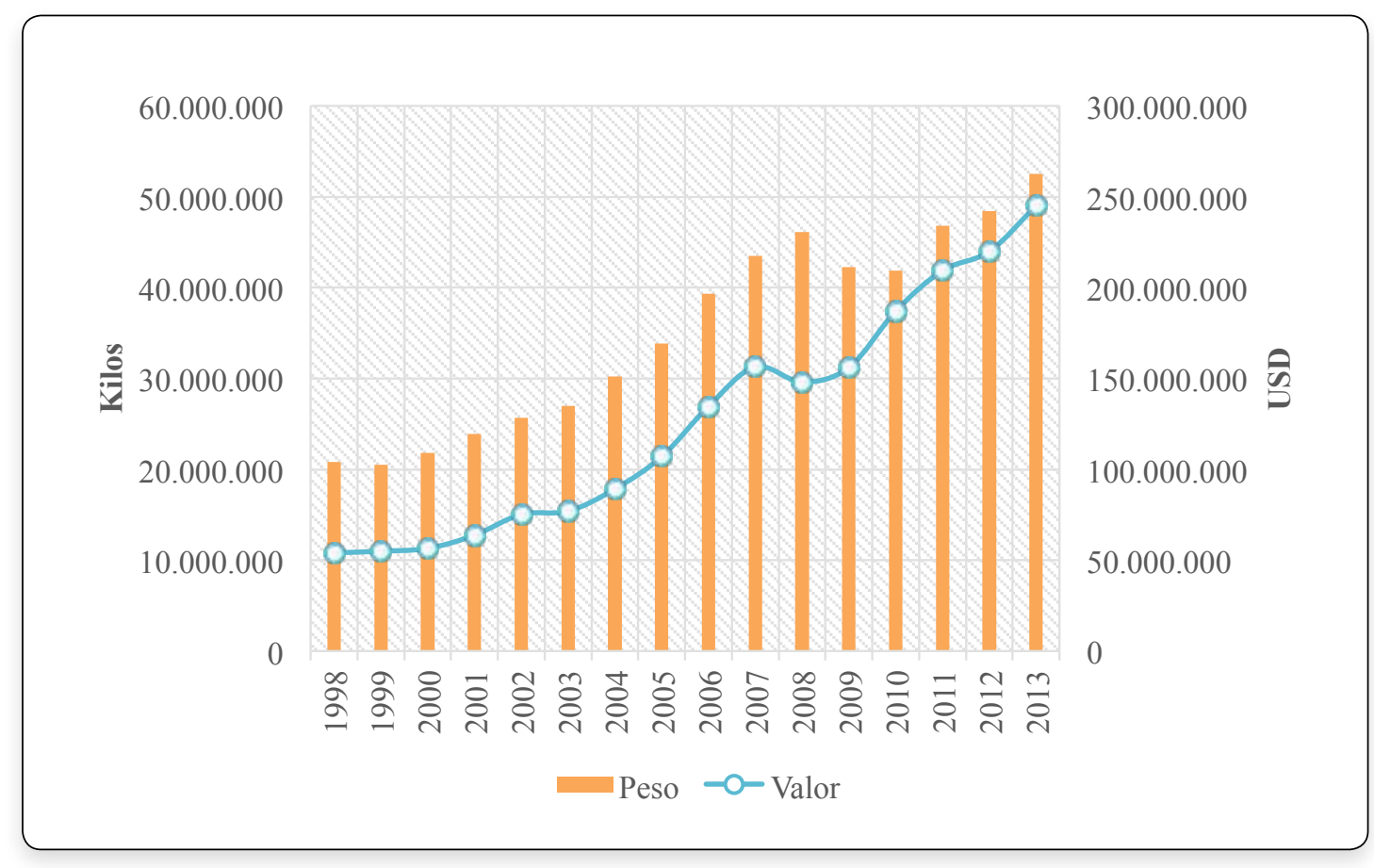

Fuente: elaboración propia con base en Dian, 2015.

En cuanto a las tendencias y evolución de la participación acumulada por país destino, la flor antioqueña se ha exportado a 91 países, y con una frecuencia constante a 35 de estos (Dian, 2015), pero con una alta concentración en Estados Unidos (91,7 \%) como principal socio, seguido de Canadá, Reino Unido y Japón, compradores que destacan como destinos que mantienen una participación sostenida en el tiempo (véase gráfica 2); en tanto que Holanda y Chile recientemente sobresalen por ser destinos de alto valor relativo en términos de valor en los últimos años, con tasas de crecimiento del 21,2 $\%$ y $55,9 \%$, respectivamente. 


\section{Gráfica 2}

Principales destinos internacionales de la flor antioqueña, 1998-2014

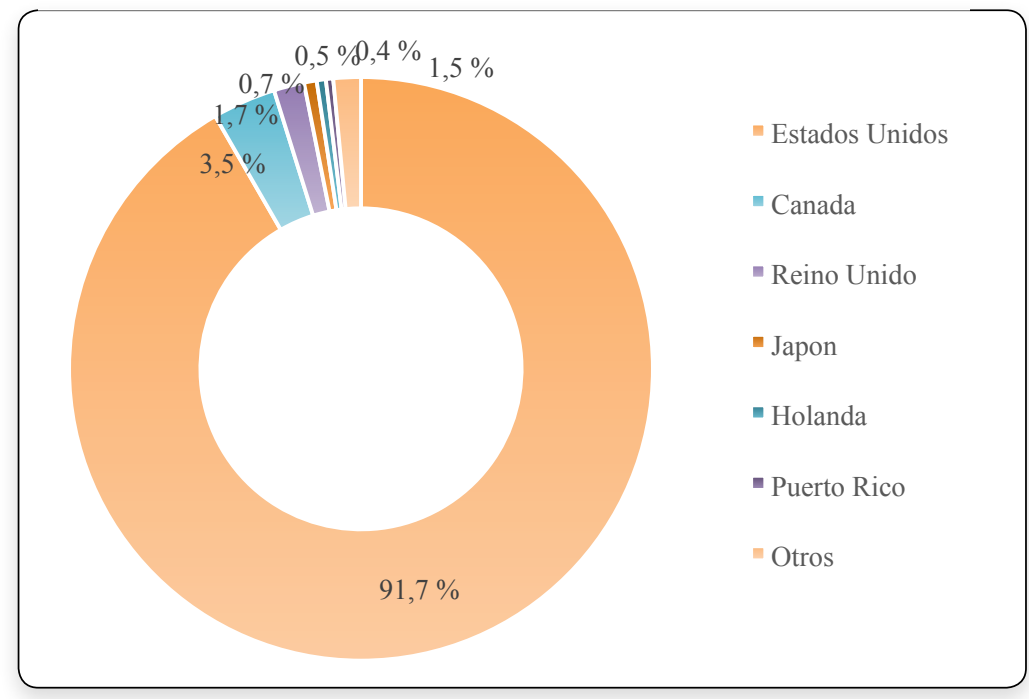

Fuente: elaboración propia con base en Dian, 2015.

En términos de los tipos de flores en el periodo 1998-2006 predominó la posición arancelaria 109000 (las demás flores y capullos frescos, cortados para ramos o adornos) con una participación del 54,2\% de la oferta exportable, seguida de las posiciones 102010 (pompones), 102000 (crisantemos) y 109400 (ramos) con un 13,2 \%, $8,1 \%$ y $7,0 \%$, respectivamente (Dian, 2015). Entre 2007 y 2014 las ventas al exterior se concentraron principalmente en las posiciones 199000 , 141000 y 149000 ; posiciones que representaron cerca del $90 \%$ del tipo de flores (véase gráfica 3).

\section{Gráfica 3}

Principales especies de flores exportables en Antioquia, 2007-2014

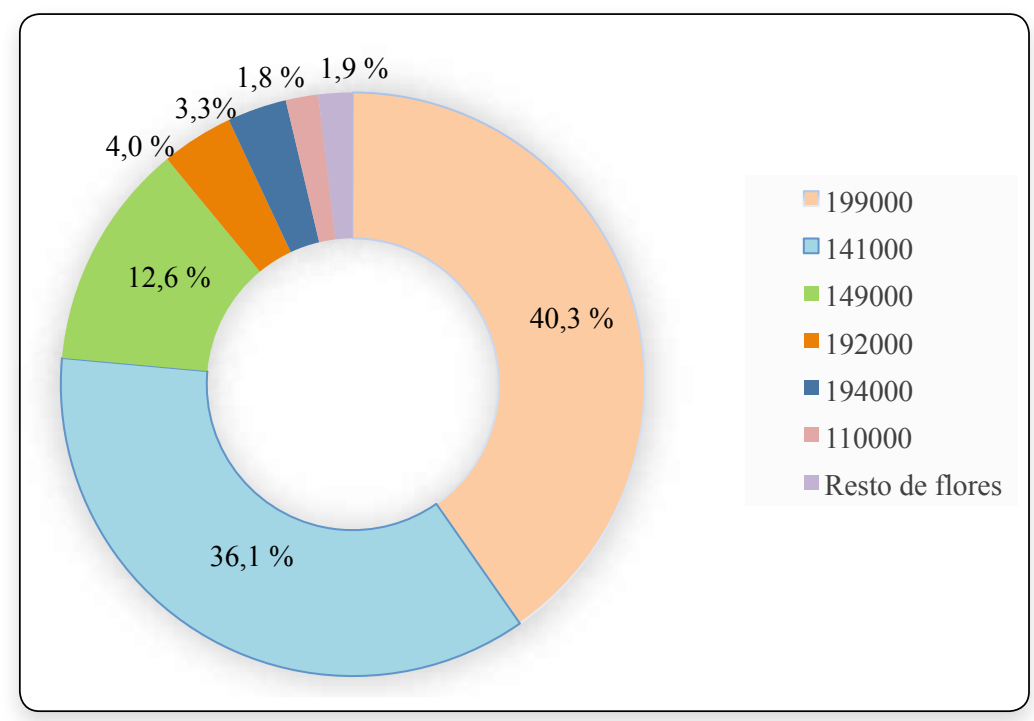

Fuente: elaboración propia con base en Dian, 2015. 
En cuanto al número de empresas, se estima que en el país 435 se dedicaron a actividades de exportación en el año 2012 (Dane, citado por González, 2013). Para el caso antioqueño los registros de la Cámara de Comercio de Medellín presentan inscritas 37 empresas en el año 2014 pertenecientes a esta actividad económica. Con base en la información del directorio exportador de la Dirección de Impuestos y Aduanas Nacionales (Dian) y la Superintendencia de Sociedades, en el periodo 2007-2014, 30 empresas antioqueñas han registrado exportaciones constantes en ese periodo, donde las ventas al exterior representan en promedio el 88 $\%$ de los ingresos operacionales (Dian, 2015; Supersociedades, 2015).

\section{Barreras a la exportación: revisión de literatura.}

Las barreras a la exportación, también denominadas problemas, obstáculos, limitantes, impedimentos, etc., hacen referencia al conjunto de factores que afectan la toma de decisiones de una empresa en el contexto internacional. Con base en lo planteado por Leonidou (1995a), las barreras a la exportación con frecuencia suscitan que muchas empresas fracasen en su aventura de posicionamiento de sus productos más allá de las fronteras nacionales, lo que no solo acarrea pérdidas financieras sino también externalidades negativas sobre las actividades presentes y futuras de otros exportadores. También se argumenta que las barreras a la exportación se pueden definir como problemas de tipo actitudinal, estructural, operacional y otra clase de restricciones que inhiben la capacidad de la firma para iniciar, desarrollar y sostener operaciones internacionales (Koksal \& Kettaneh, 2011).

La revisión de la literatura académica sugiere una diversidad de aproximaciones al problema de los factores que impiden la expansión exportadora. A continuación se presentan los casos de estudio para Colombia, una síntesis en el ámbito internacional y los estudios que se enfocan específicamente en el sector floricultor.

\section{Barreras a la exportación en Colombia.}

Para el caso colombiano varios trabajos plantean los obstáculos que deben sortear las empresas al momento de internacionalizarse vía exportaciones. Puyana (2004) presenta la baja capacidad administrativa para vinculación con el sector externo como un factor inhibidor común en las pymes colombianas, además de la poca información sobre oportunidades para exportar de forma competitiva y sostenible, con productos que contengan alto valor agregado local. También la mínima capacitación y gestión gerencial en el área internacional y el limitado acceso a tecnologías, en especial las que atañen al comercio exterior.

Los mayores obstáculos al proceso exportador de las pymes nacionales subyacen en factores relacionados con el marketing, a saber: la adecuación de los productos a requerimientos del mercado externo, la falta de información y conocimiento de los mercados, el acceso a canales de distribución adecuados y la poca capacitación en marketing internacional.

Carazo (2007, 2009) se enfoca en la promoción del proceso de desarrollo exportador para pymes del Caribe colombiano, encontrando que los factores internos, como la carencia de recursos y capacidades organizacionales y directivas, son recurrentes a la hora de una firma permanecer en los mercados internacionales. Por su parte, Escandón, Ayala y Caicedo (2013) asocian las barreras y el compromiso exportador, evidenciando que el grupo de barreras financieras-legales reporta una incidencia considerable en esa relación, la cual está mediada por los altos costos financieros, desconocimiento de beneficios financieros, estándares técnicos, normativas de seguridad y exigencias al producto (etiquetas, presentación). 


\section{Perspectivas internacionales a los obstáculos a las exportaciones.}

Dentro del grupo de trabajos que capturan la percepción de los agentes económicos hay un conjunto amplio de enfoques. Así, por ejemplo, Leonidou (1995b) para empresas no exportadoras encuentra cómo las crecientes presiones competitivas son el más fuerte impedimento. Este mismo autor en una investigación posterior detalla que el tamaño de la empresa, la experiencia y la creación de redes de respaldo son los problemas que ejercen el mayor impacto (Leonidou, 2000).

En el caso de Da Silva y Da Rocha (2001) esos problemas se ligan al tipo de industria, el tamaño de la empresa, la experiencia exportadora y el alcance geográfico de la exportación. Suárez-Ortega (2003) halló cuatro determinantes de las barreras: diferencias relativas al marketing, información relativa al mercado y el proceso exportador, recursos internos y de personal limitados y algunos obstáculos del entorno. Patterson (2004) subraya que los asuntos relacionados con el compromiso y la preparación para invertir, la falta de experiencia en marketing internacional y de contactos extranjeros, así como el grado de intensidad competitiva son los principales obstáculos.

Por su parte, Smith, Gregoire y Lu (2006) señalan que concurren diferencias en las percepciones de los gerentes de firmas que exportan y que no exportan, así como la presencia de distintas etapas de exportación y proceso de desarrollo. El trabajo de Villavicencio (2007) expone las habilidades gerenciales, estrategias de mercadotecnia y condiciones financieras. En el caso de Pérez y Camarero (2007) la actitud proactiva o reactiva frente a la exportación configuran factores que ayudan a explicar la diferencia de percepciones. Entretanto, Arteaga-Ortiz y Fernández-Ortiz (2010) confirman cuatro dimensiones o factores de barreras a la exportación, a saber: el conocimiento, los recursos, el procedimiento y las barreras exógenas.
Koksal y Kettaneh (2011) indican como limitantes internas la capacidad de producción, el sistema de empaque y los costos del proceso exportador, mientras que las externas fueron las barreras arancelarias y no arancelarias. Jalali (2012) muestra varias dimensiones de análisis en el problema de las barreras: operativa, ambiental, financiera, origen, legal y logística. Milanzi (2012) sostiene que es más probable encontrar menos barreras a exportar mientras mayor sea el número de redes que se consolidan. Otro factor que destaca Uner, Kocak, Cavusgil y Cavusgil (2013) es que el estadio de internacionalización que alcanzan las firmas condiciona el desempeño exportador.

Arteaga-Ortiz, Fernández-Ortiz y San Emeterio (2014) contrastan la importancia relativa atribuida a las diversas barreras a la exportación y su variación en función del destino de las exportaciones de la empresa. Recientemente, Ayob, Ramlee y Rahman (2015) explicaron cómo los factores financieros profesan un papel importante en la internacionalización de las empresas; sin embargo, no existe claridad alrededor del impacto de factores como el costo y el capital y de su relación con el comportamiento exportador.

Ahora bien, complementando la revisión anterior con estados del arte en el tema, se dispone de un amplio grupo de trabajos de barreras a la exportación, que pueden agrupar los factores inherentes al desempeño de la compañía (internos) y los relativos al entorno natural de esta (externos) (Kaleka \& Katsikeas, 1995; Leonidou, 1995a; Tesfom \& Lutz, 2006) (véase cuadro 1). Otros autores distinguen entre problemas externos, operacionales, internos e informacionales (Katsikeas \& Morgan, 1994); entre limitantes empresariales e industriales (Tesfom \& Lutz, 2006); así como entre factores objetivos o actuales y subjetivos o percibidos (Doern, 2009). Una clasificación más sistémica fue propuesta por Leonidou (1995a), al desagregarlos en un esquema de cuatro categorías: a) internos domésticos, b) externos 
domésticos, c) internos internacionales y d) externos internacionales.

\section{Barreras en el sector floricultor.}

En el caso concreto de barreras a la exportación del sector floricultor existen algunos estudios aplicados. Uno de estos aborda las barreras percibidas a las exportaciones específicas para la industria de la horticultura de Australia y su impacto relativo sobre las decisiones de exportación de las empresas; se sugieren siete factores principales que la restringen. Las barreras identificadas difieren de aquellas plasmadas en estudios donde se clasifican como propias de la industria o del país. Por otro lado, la percepción de impacto de algunos de los obstáculos fue significativamente distinta entre las empresas no exportadoras y las exportadoras (Ramaseshan \& Soutar, 1995).

\section{Cuadro 1}

Principales barreras a las exportaciones según diversas revisiones literarias

\begin{tabular}{|c|c|}
\hline Barreras internas & Barreras externas \\
\hline $\begin{array}{l}\text { - Mercadeo y conocimiento: } \\
\text { Estrategias, mercados y procedimientos de exportación, } \\
\text { experiencia, frecuencia de exportación, identificación } \\
\text { de clientes, contratos y comunicación, representantes } \\
\text { internacionales y confianza }\end{array}$ & $\begin{array}{l}\text { Industria: } \\
\text { Estructura, precios, competencia, concentración, tecnolo- } \\
\text { gía, materias primas y rentabilidad }\end{array}$ \\
\hline $\begin{array}{l}\text { Financieras: } \\
\text { Capital de trabajo, financiación, presupuesto, pagos, } \\
\text { tamaño de la empresa, costos iniciales, crédito y ventas }\end{array}$ & $\begin{array}{l}\text { Mercado: } \\
\text { Demanda, imagen, cultura e idioma, familiaridad, pagos, } \\
\text { precios (tasa de cambio e interés), costos, transporte e } \\
\text { infraestrutura }\end{array}$ \\
\hline $\begin{array}{l}\text { - Recurso humano: } \\
\text { Gestión, entrenamiento y capacidades y toma de deci- } \\
\text { siones }\end{array}$ & $\begin{array}{l}\text { Legales: } \\
\text { Documentación y procedimientos, aduanas, normas de } \\
\text { origen, regulación, asistencia, políticas y licencias }\end{array}$ \\
\hline $\begin{array}{l}\text { Organización: } \\
\text { Tipo de productos, calidad, imagen, insumos, empa- } \\
\text { quetado y etiquetas, logística, diseño y especificación y } \\
\text { diversificación }\end{array}$ & \\
\hline
\end{tabular}

Fuente: elaboración propia con base en Leonidou, 1995a; 2000; Kaleka y Katsikeas, 1995; Tesfom y Lutz, 2006; y Kahiya, 2013.

En otro contexto, Belwal y Chala (2008) formulan una evaluación ambiental de la industria de la floricultura en Etiopía, para desvelar los catalizadores y barreras que prevalecen en la industria. En síntesis, las amenazas percibidas residen en los cuellos de botella en infraestructura, la escasez de insumos agrícolas, la limitada gama de productos y la ausencia de adhesión a los códigos internacionales de buenas prácticas; no obstante, en su conjunto, existe una tendencia creciente en el desarrollo de la industria de la floricultura en Etiopía, que atrae inversores nacionales y extranjeros apalancada por la atención del Gobierno y por las ventajas comparativas de este país.

Un última investigación identifica los factores que influyen en la intensidad del éxito de exportación en Ghana (Egyir, Mensah, \& AgyeiSasu, 2012); encontrando que dicha intensidad se liga con el nivel educativo del gerente, la experiencia, la formación y la orientación hacia el emprendimiento, así como la presencia de un departamento de exportación, la diversificación de productos y el apoyo del Gobierno influyen directamente en la intensidad de las barreras y del éxito en la exportación. Como principal 
limitación, sugieren el capital de trabajo como agente negativo en la intensidad del éxito de las exportaciones.

\section{Materiales y métodos}

Esta investigación siguió una metodología mixta. Por un lado, se capturó la percepción de expertos alrededor de las barreras que enfrentan las empresas floricultoras en Antioquia. Por otro, apoyados en la técnica de proceso de análisis jerárquico (AHP, por su sigla en inglés), se categorizan los obstáculos, se organizan en orden de importancia relativa y se determina el nivel de impacto de estos para los principales destinos de exportación. La recolección de información se guio por entrevistas estructuradas a una muestra no aleatoria intencionada de seis expertos, consistente de dos gerentes de compañías, tres directivos de gremios y un académico.

\section{Etapas.}

Las etapas que se ejecutaron para alcanzar el objetivo propuesto fueron:

1. Identificación de obstáculos con base en la revisión de literatura académica. Con esta información se crearon las categorías de análisis para desarrollar el ejercicio de valoración.

2. Definición de la estructura jerárquica en un mundo AHP, considera objetivo global, criterios, subcriterios y alternativas. En este sentido, se propusieron cinco niveles jerárquicos: a) objetivo: valoración de factores que afectan el comercio internacional de productos de plantas vivas y productos de floricultura; b) dimensiones estratégicas: barreras internas y externas, c) criterios: recurso humano, organización, mercado y legislación; d) subcriterios: falta de visión gerencial, personal poco calificado, costos de transporte y dificultades logísticas, revaluación de la moneda, falta de estudios o conocimiento de mercados para exportar, infraestructura empresarial, altos costos de financiación para exportar (costos de transacción y costos ocultos), competencia internacional, restricciones internacionales y reglas de país destino, y trámites burocráticos y trabas legales; y e) alternativas: principales mercados destino del producto, Estados Unidos, Canadá, Reino Unido y Japón.

3. Recolección, medición y procesamiento de datos. Una vez definida la jerarquía, se diseñó un formulario de recolección de información que los expertos diligenciaron. En Microsoft Excel se programaron los sistemas matriciales para arribar a las soluciones y síntesis de resultados. Para resolver los sistemas se normalizaron los vectores y la solución única se alcanza mediante valores y vectores propios que hacen máxima la solución (Saaty, 1994).

Las comparaciones pareadas siguieron la escala fundamental de nueve puntos propuesta por Saaty (1994). Se llegó a un resultado global agrupando las preferencias individuales de cada experto (Forman \& Peniwati, 1998) empleando la media geométrica (Aczél \& Saaty, 1983). Para el último nivel de la jerarquía se adaptó la escala Liberatore (1987) en tres alternativas de valoración: obstáculos altos a la exportación, obstáculos medios a la exportación y obstáculos bajos a la exportación.

\section{Modelo proceso de análisis jerárquico.}

La estructura jerárquica AHP es una de las aproximaciones metodológicas más utilizada para priorizar factores (Saaty \& Peniwati, 2013). Esta herramienta parte de una teoría de jerarquización que deriva escalas relativas de números absolutos desde juicios de valor o percepciones expresadas en una escala fundamental numérica en un rango de 1 a 9. Estas valoraciones se consignan en un arreglo matricial cuadrado de orden $n x n$, con diagonal principal unitaria y con diagonal inferior que toma el valor recíproco de la diagonal superior. Matemáticamente, el sistema matricial que se conforma se expresa tal como se muestra en la ecuación (1) (Saaty, 1994). 


$$
\mathrm{A}=\underset{A n}{A 1}\left[\begin{array}{ccc}
a_{11} & \cdots & a_{1 n} \\
\vdots & \ddots & \vdots \\
a_{n 1} & \cdots & a_{n n}
\end{array}\right]
$$

$W w=n w(W-n I) w=0$, donde $w_{i} / w_{j}$ se aproxima mediante $a_{i j}=w_{i} / w_{j}$ y $a_{j i}=1 / a_{i j}$ y $a_{i i}=1$. De esta forma:

$$
A=\left[a_{i j}\right] n x n
$$

El sistema de ecuaciones se hace máximo mediante el método de valores y vectores propios (ecuación 3), por lo que:

$$
A w=n w \Rightarrow\left(A-\gamma_{\max } I\right) w=0
$$

De esta manera, se puede validar la bondad de ajuste del sistema matricial (ecuación 4) mediante un índice de consistencia (IC):

$$
I C=\left(\gamma_{\max }-n\right) /(n-1)
$$

Los juicios expresados por cada individuo, $a_{i j}^{k}, k=1, \ldots, m$, se agregaron para obtener una representación global de los datos mediante la media geométrica (ecuación 5), así:

$$
a_{i j}^{G l o b a l}=\prod_{k=1}^{m}\left(a_{i j}^{k}\right)^{\alpha_{k}}
$$

\section{Estructura de valoración propuesta.}

Con base en la revisión literaria (Leonidou, 1995a, 2000; Tesfom \& Lutz, 2006; Kahiya, 2013) y en el contexto de análisis, este estudio adapta algunas de las barreras identificadas e incluye otras que son particularmente aplicables al sector floricultor. Las limitantes se clasifican en dos categorías: internas y externas, las cuales se subdividen en otras subcategorías que permiten evaluarlas por separado y después para los principales destinos de exportación del sector. La estructura jerárquica de valoración diseñada sigue el siguiente patrón (véase figura 1).

Así, para el caso de estudio se tendrán cada una de las categorías a evaluar $\left(A_{p}, \ldots, A_{n}\right)$ y sus respectivas ponderaciones $\left(W_{l}, \ldots, W_{n}\right)$. Luego, los expertos compararán cada alternativa $A_{i}$ con la alternativa $A_{j}$, mediante la asignación de un valor numérico $a_{i j}$ que representa la intensidad de la importancia de $A_{i}$ sobre $A_{j}$ El valor $a_{i j}$ es una aproximación de la relevancia relativa de las categorías comparadas, $a_{i j}=$ $W / W_{j}$. Por ejemplo, la figura 1 muestra que dentro de los factores organizacionales se identificaron tres barreras $\left(A_{1}, A_{2}, A_{3}\right)$ : $\mathrm{A}_{1}$ : infraestructura empresarial, $\mathrm{A}_{2}$ : conocimiento de mercados y $\mathrm{A}_{3}$ : transporte y logística; por lo que se conforma una matriz de orden $3 \mathrm{x}$ 3 con las comparaciones realizadas por cada experto, donde la posición $a_{13}$ corresponde a la importancia relativa de la infraestructura empresarial sobre el transporte y logística como obstáculo determinante de las exportaciones del sector en cuestión.

El mismo procedimiento se sigue para cada una las categorías identificadas en la figura 1, dando lugar a la resolución de siete sistemas matriciales por cada experto consultado, cada uno de diferente dimensión dependiendo del número de criterios a evaluar. En el anexo 1 se presentan las comparaciones realizadas que dan paso a los sistemas de ecuaciones a resolver y agregar para arribar a una solución global. 
Figura 1

Estructura jerárquica de valoración de factores

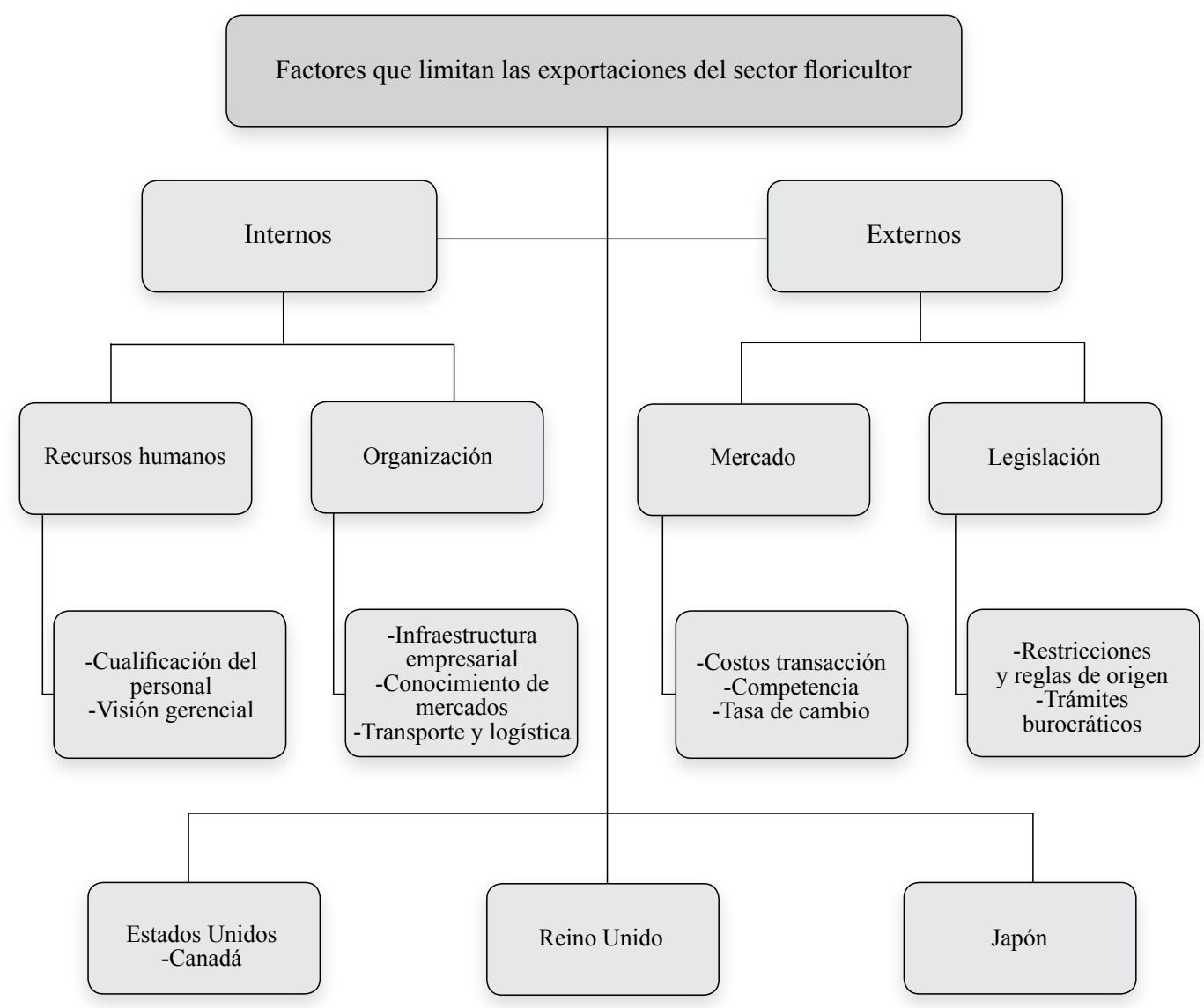

Fuente: elaboración propia.

\section{Análisis empírico}

Con base en las entrevistas en profundidad y la aplicación de la metodología multicriterio AHP, la percepción acerca de las principales limitantes que encara el sector floricultor se muestra en la figura 2. Como se puede observar, los ratios presentados allí corresponden a la agregación geométrica de los juicios de valor emitidos por los expertos participantes del estudio. En los principales obstáculos que enfrenta esta actividad económica destacan los factores internos a las empresas $(0,842)$; y dentro de esta categoría de análisis el mayor peso recae sobre el capital humano $(0,691)$.

Una vez se desagregan las subbarreras, los pesos globales dan cuenta de que los principales limitantes percibidos son la falta de visión gerencial $(0,319)$, la existencia de personal poco calificado a la hora de afrontar la negociación internacional $(0,263)$ y las dificultades organizacionales en cuanto a los costos logísticos y de transporte $(0,134)$. Así mismo, el principal problema de índole externo que se percibe se asocia a la volatilidad de la divisa $(0,072)$. 
Figura 2

Resultados globales de la valoración de barreras, sub barreras y destinos

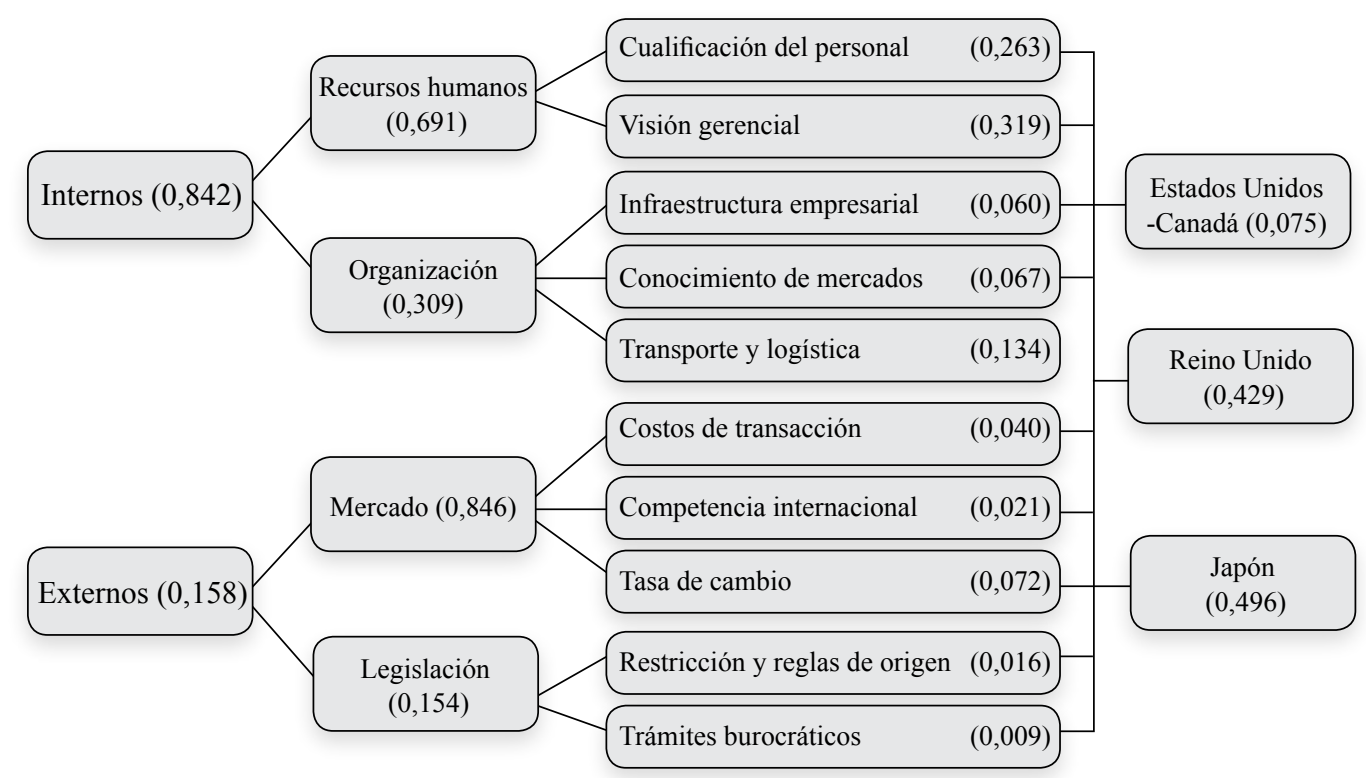

Fuente: elaboración propia.

Desde una perspectiva crítica, los resultados dejan ver que los activos intangibles con que cuenta una compañía pueden obstaculizar el desarrollo de su futuro exportador (véase gráfica 4). Luego, por un lado, estos resultados soportan los hallazgos de estudios previos que señalan que el compromiso de la dirección de una empresa, así como el entrenamiento del personal, restrin- gen el desarrollo de actividades en los mercados internacionales (Kaleka \& Katsikeas, 1995), siendo crítica la dependencia de las actitudes y características de los gerentes (Tesfom \& Lutz, 2006). Así, el éxito exportador pasa por los tomadores de decisiones que son responsables del diseño de estrategias, que incluye el posicionamiento de productos en el exterior.

\section{Gráfica 4}

Priorización de barreras a la exportación

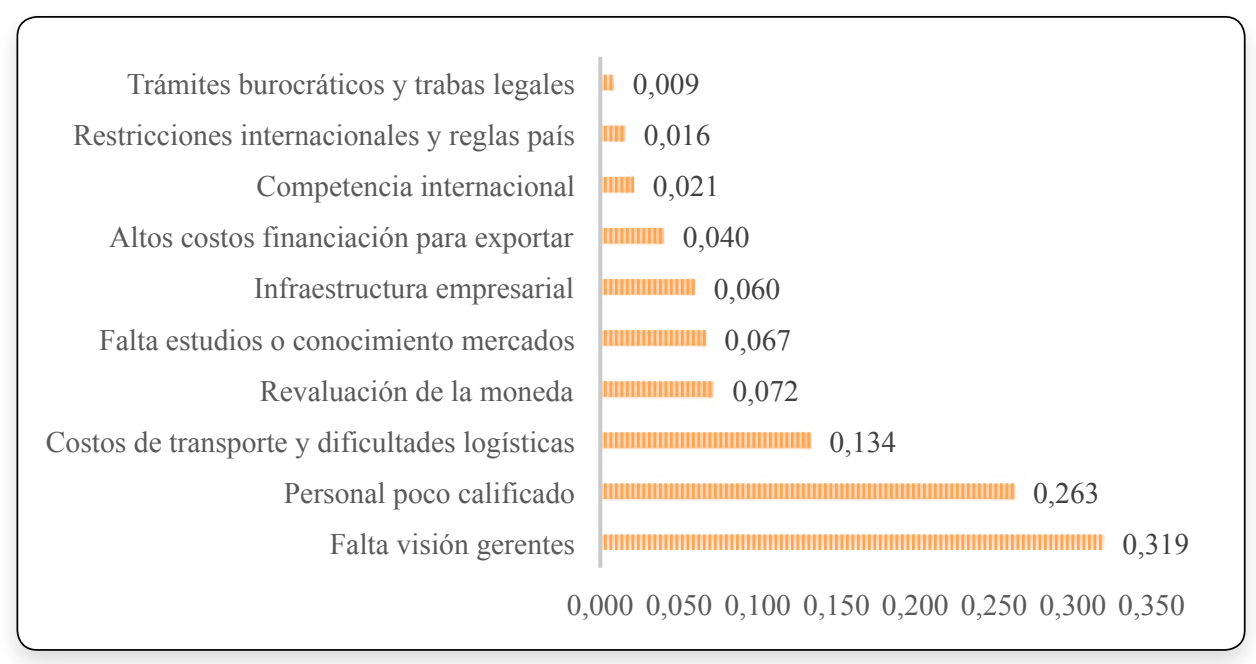

Fuente: elaboración propia. 
Un resultado destacable es que la posición de los distintos actores, desde el punto de vista de la demanda (empresario), así como desde la óptica de la oferta institucional (gremios y entidades del Estado), coincide en evidenciar que la visión gerencial puede inhibir la exploración o desarrollo de futuras oportunidades de negocio. Sin lugar a dudas, los factores que afectan una empresa son de corte multidimensional, razón por la cual los asuntos organizacionales y las fluctuaciones cambiarias se perciben como otras de las limitantes que afectan el sector.

Por último, al comparar la percepción sobre el grado de presencia de obstáculos en los principales destinos de exportación de flores se encuentra que los destinos geográficamente más alejados, como Japón y Reino Unido, tienen un nivel más alto percibido de obstáculos que los países más cercanos como Estados Unidos y Canadá.

\section{Conclusiones y limitaciones}

El propósito de este artículo fue identificar y evaluar los factores que inhiben el posicionamiento de los productos exportables del sector floricultor antioqueño en los mercados internacionales, en particular Estados Unidos, Canadá, Reino Unido y Japón. La investigación revela cómo la escasa visión gerencial limita fuertemente la consolidación y expansión de este tipo de productos, seguida del recurso humano especializado y los costos de transporte y la logística. El diagnóstico del sector floricultor, calificado a través de la percepción de empresarios y gremios, permitió jerarquizar los factores de acuerdo con su importancia relativa y cuantificar su impacto para los principales mercados destino de exportación.

El aporte capital de este trabajo consiste en suministrar elementos de juicio a la discusión y el análisis, tanto para que las instituciones de apoyo a la promoción y apertura de nuevos mercados como los directamente implicados -gremios y empresarios- diseñen estrate- gias para el apoyo y la promoción exportadora nacional. La principal limitación subyace en la carencia de un registro sistemático de información histórica, no solo en empresas del sector floricultor sino para el parque industrial en general, razón por la que se recurre a estudios de corte no paramétrico, para determinar las limitaciones del proceso exportador.

\section{Referencias}

Aczél, J., \& Saaty, T. (1983). Procedures for synthesizing ratio judgements. Journal of mathematical Psychology, 27(1), 93-102.

Arteaga-Ortiz, J., \& Fernández-Ortiz, R. (2010). Why Don't We Use the Same Export Barrier Measurement Scale? An Empirical Analysis in Small and Medium-Sized Enterprises. Journal of Small Business Management, 48(3), 395-420.

Arteaga-Ortiz, J., Fernández-Ortiz, R., \& San Emeterio, M. (2014). Las pymes españolas en Latinoamérica: barreras a la exportación. Revista AD-Minister, 24, 53-78.

Asocolflores. (2009). Segunda semana de la competitividad de Asocolflores (Asocolflores, Documento presentado como análisis del sector floricultor). Bogotá: Autor.

Asocolflores. (2015). Boletín Estadístico 2015. Bogotá: Autor.

Ayob, A., Ramlee, S., \& Rahman, A. (2015). Financial factors and export behavior of small and medium-sized enterprises in an emerging economy. Journal of International Entrepreneurship, 13(1), 49-66. doi: 10.1007/s10843-014-0141-5

Belwal, R., \& Chala, M. (2008). Catalysts and barriers to cut flower export: A case study of Ethiopian floriculture industry. International Journal of Emerging Markets, 3(2), 216-235. 
Calderón, M. (2014). Análisis de eficiencia técnica y estudio de casos en los cultivos de flores de la Sabana de Bogotá. Pensamiento \& Gestión, 36, 291-326.

Carazo, P. (2007). Influencia de la promoción de exportaciones en el proceso del desarrollo exportador de las Pymes. Un estudio de caso. Pensamiento \& Gestión, 23, 1-57.

Carazo, P. (2009). Proceso del desarrollo exportador de la pyme colombiana. Cuadernos de Gestión, 9(1), 47-66.

Cavusgil, S., \& Kirpalani, V. (1993). Introducing products into export markets: success factors. Journal of Business Research, 27(1), 1-15.

Da Silva, P., \& Da Rocha, A. (2001). Perception of export barriers to Mercosur by Brazilian firms. International Marketing Review, 18(6), 589-611.

Dirección de Impuestos y Aduanas Nacionales -[DIAN]. (2015). Sistema Estadístico de Comercio Exterior. Recuperado de http:// websiex.dian.gov.co/

Doern, R. (2009). Investigating barriers to SME growth and development in transition environments: A critique and suggestions for developing the methodology. International Small Business Journal, 27(3), 275-305.

Egyir, I., Mensah, E., \& Agyei-Sasu, F. (2012). Factors influencing the intensity of export success in Ghana's horticultural industry. International Journal of Business, Humanities and Technology, 2(7), 115-120.

Escandón, D., Ayala, A., \& Caicedo, M. (2013). Influencia de las barreras a la exportación sobre el compromiso exportador y su incidencia en los resultados internacionales. Revista EAN, 75, 38-55.
Forman, E., \& Peniwati, K. (1998). Aggregating individual judgments and priorities with the analytic hierarchy process. European Journal of Operational Research, 108(1), 165-169.

González, A. (2013). Intercambio de información en las cadenas de suministro internacionales. El caso de la cadena de suministro de flor fresca cortada colombiana para la exportación (CEPAL, Serie Comercio Internacional 120). Santiago de Chile: Naciones Unidas.

Jalali, S. (2012). Export Barriers and Export Performance: Empirical Evidence from the Commercial Relationship between Greece and Iran. South-Eastern Europe Journal of Economics, 10(1), 53-66.

Kahiya, E. (2013). Export barriers and path to internationalization: A comparison of conventional enterprises and international new ventures. Journal of International Entrepreneurship, 11(1), 3-29.

Kaleka, A., \& Katsikeas, S. (1995). Exporting problems: the relevance of export development. Journal of Marketing Management, 11, 499-515.

Katsikeas, C., \& Morgan, R. (1994). Differences in Perceptions of Exporting Problems Based on Firm Size and Export Market Experience. European Journal of Marketing, 28(5), 17-35.

Koksal, M., \& Kettaneh, T. (2011). Export problems experienced by high-and lowperforming manufacturing companies: A comparative study. Asia Pacific Journal of Marketing and Logistics, 23(1), 108-126.

Leonidou, L. (1995a). Empirical research on export barriers: review, assessment, and synthesis. Journal of International Marketing, 3(1), 29-43. 
Leonidou, L. (1995b). Export barriers: nonexporters' perceptions. International Marketing Review, 12(1), 4-25.

Leonidou, L. (2000). Barriers to export management: an organizational and internationalization analysis. Journal of International Management, 6(2), 121-148.

Liberatore, M. (1987). An extension of the analytic hierarchy process for industrial $\mathrm{R} \& \mathrm{D}$ project selection and resource allocation. Engineering Management, IEEE Transactions on, 34(1), 12-18.

Milanzi, M. (2012). Export barrier perceptions in Tanzania: The influence of social networks. Journal of African Business, 13(1), 29-39.

Patterson, P. (2004). A study of perceptions regarding service firms' attitudes towards exporting. Australasian Marketing Journal, 12(2), 19-38.

Pérez, F., \& Camarero, L. (2007). Intensidad exportadora y percepción de barreras a la exportación: un estudio de casos. Investigaciones europeas de dirección y economía de la empresa, 13(3), 93-106.

Puyana, D. (2004). La pyme y su situación en Colombia. Revista Civilizar Ciencias Sociales y Humanas, 6, 63-81.

Quirós, M. (2001). La Floricultura en Colombia en el marco de la globalización: Aproximaciones hacia un análisis micro y macroeconómico. Revista Universidad EAFIT, 37(122), 59-68.

Ramaseshan, B., \& Soutar, G. (1995). Export Barriers in the Western Australian Horticulture Industry. Journal of International Food \& Agribusiness Marketing, 6(4), 53-70.
Reina, M., \& Acosta, P. (2008). El sector floricultor frente a la revaluación: situación actual y alternativas de política. Fundación para la educación superior y el desarrollo. Bogotá: Fundación para la Educación Superior y el Desarrollo. Recuperado de http://www.repository.fedesarrollo.org.co/ bitstream/11445/1041/1/Repor_Noviembre 2008 Reina Acosta y_Oviedo.pdf

Saaty, T. (1994). Fundamentals of decision making and priority theory with the analytic hierarchy process (The Analytic Hierarchy Process Series, vol.6). Pittsburgh: RWS Publications.

Saaty, T., \& Peniwati, K. (2013). Group decision making: drawing out and reconciling differences. Pittsburgh: RWS publications.

Smith, D., Gregoire, P., \& Lu, M. (2006). Managers' Perceptions of Export Barriers: A CrossCultural Perspective of Service Firms. Journal of Transnational Management, 12(1), 51-68.

Suárez-Ortega, S. (2003). Export Barriers: Insights from Small and Medium Sized Firms. International Small Business Journal, 21(4) 403-419.

Superintendencia de Sociedades - [Supersociedades]. (2015). Sistema de Información y Riesgo Empresarial (SIREM), 20072014. Recuperado de http://sirem.supersociedades.gov.co/Sirem2/

Tesfom, G., \& Lutz, C. (2006). A classification of export marketing problems of small and medium sized manufacturing firms in developing countries. International Journal of Emerging Markets, 1(3), 262-281.

Uner, M., Kocak, A., Cavusgil, E., \& Cavusgil, S. (2013). Do barriers to export vary for born globals and across stages of internationalization? An empirical inquiry in the emerging 
market of Turkey. International Business Review, 22(5), 800-813.

Villavicencio, J. (2007). Factores que inciden en el desempeño exportador de las Pymes: una aplicación empírica. Tec Empresarial, 1(4), 28-40.

Zafarullah, M., Mujahid, A., \& Young, S. (1998). The internationalization of the small firm in developing countries - exploratory research from Pakistan. Journal of Global Marketing, 11(3), 21-40. 


\section{Anexo 1}

\section{Categorías de análisis, variables e instru- mento de recolección de información}

Diferentes estudios nacionales e internacionales han demostrado la presencia de barreras y obstáculos que reportan las empresas a la hora de posicionar sus productos en los mercados internacionales. A continuación encontrará las principales barreras que hemos identificado en el contexto del sector floricultor. El objetivo es que usted como experto y desde su experiencia compare dos alternativas según su importancia y las califique (valore) en una escala de 1 a 9. Los resultados serán relevantes para el desarrollo de un proyecto de investigación que adelanta el Tecnológico de Antioquia Institución Universitaria.

1. ¿Cuál de los siguientes obstáculos cree usted que incide más a la hora de una empresa del sector floricultor decida exportar? Compare cada una de las parejas de obstáculos, marque con una $\mathrm{X}$ el que considere más importante $\mathrm{y}$ califique esa importancia en una escala de 1 a 9 .

\begin{tabular}{|c|c|c|}
\hline Obstáculo X & Obstáculo Y & Valoración \\
\hline Interno & Externo & \\
\hline Recursos humanos & Organizacional & \\
\hline Mercado & Legislación & \\
\hline
\end{tabular}

2. Compare y valore cada una de las subcategorías de obstáculos a las exportaciones con el fin de establecer cuáles son los más significativos dentro de cada grupo. Coteje cada uno de los pares de ellas, marque con una $\mathrm{X}$ el que considere más importante y califique esa importancia en una escala de 1 a 9.

\begin{tabular}{|c|c|c|}
\hline Obstáculo X & Obstáculo Y & Valoración \\
\hline \multicolumn{3}{|c|}{ Recursos humanos } \\
\hline $\begin{array}{l}\text { Falta de personal } \\
\text { calificado }\end{array}$ & $\begin{array}{l}\text { Falta de visión } \\
\text { empresarial }\end{array}$ & \\
\hline \multicolumn{3}{|c|}{ Organizacionales } \\
\hline $\begin{array}{l}\text { Infraestructura } \\
\text { empresarial }\end{array}$ & $\begin{array}{l}\text { Falta de estudios } \\
\text { o conocimiento } \\
\text { de mercados para } \\
\text { exportar }\end{array}$ & \\
\hline
\end{tabular}

\begin{tabular}{|c|c|c|}
\hline Obstáculo X & Obstáculo Y & Valoración \\
\hline $\begin{array}{l}\text { Infraestructura } \\
\text { empresarial }\end{array}$ & $\begin{array}{l}\text { Costos de } \\
\text { transporte y } \\
\text { dificultades } \\
\text { logísticas }\end{array}$ & \\
\hline $\begin{array}{l}\text { Falta de estudios o } \\
\text { conocimiento de } \\
\text { mercados para } \\
\text { exportar }\end{array}$ & $\begin{array}{l}\text { Costos de } \\
\text { transporte y } \\
\text { dificultades } \\
\text { logísticas }\end{array}$ & \\
\hline & Mercado & \\
\hline $\begin{array}{l}\text { Altos costos de } \\
\text { financiación para } \\
\text { exportar }\end{array}$ & $\begin{array}{l}\text { Competencia } \\
\text { internacional }\end{array}$ & \\
\hline $\begin{array}{l}\text { Altos costos de } \\
\text { financiación para } \\
\text { exportar }\end{array}$ & $\begin{array}{l}\text { Revaluación de la } \\
\text { moneda }\end{array}$ & \\
\hline \multirow[t]{2}{*}{$\begin{array}{l}\text { Competencia } \\
\text { internacional }\end{array}$} & $\begin{array}{l}\text { Revaluación de la } \\
\text { moneda }\end{array}$ & \\
\hline & Legislación & \\
\hline $\begin{array}{l}\text { Restricciones } \\
\text { internacionales y } \\
\text { reglas país destino }\end{array}$ & $\begin{array}{l}\text { Trámites } \\
\text { burocráticos y } \\
\text { trabas legales }\end{array}$ & \\
\hline
\end{tabular}

3. ¿Cuáles de los siguientes destinos de exportación cree usted que son más propensos a presentar los riesgos identificados anteriormente? En este caso tiene tres alternativas de valoración: obstáculos altos a la exportación (A), obstáculos medios a la exportación (M) y obstáculos bajos a la exportación (B).

\begin{tabular}{|c|c|c|c|}
\hline Obstáculo/destino & $\begin{array}{l}\text { Estados } \\
\text { Unidos- } \\
\text { Canadá }\end{array}$ & $\begin{array}{l}\text { Reino } \\
\text { Unido }\end{array}$ & Japón \\
\hline Personal poco calificado & & & \\
\hline Falta de visión gerentes & & & \\
\hline $\begin{array}{l}\text { Infraestructura } \\
\text { empresarial }\end{array}$ & & & \\
\hline $\begin{array}{l}\text { Falta de estudios o } \\
\text { conocimiento de } \\
\text { mercados para exportar }\end{array}$ & & & \\
\hline $\begin{array}{l}\text { Costos de transporte y } \\
\text { dificultades logísticas }\end{array}$ & & & \\
\hline $\begin{array}{l}\text { Altos costos de } \\
\text { financiación para exportar }\end{array}$ & & & \\
\hline $\begin{array}{l}\text { Competencia } \\
\text { internacional }\end{array}$ & & & \\
\hline Revaluación de la moneda & & & \\
\hline $\begin{array}{l}\text { Restricciones } \\
\text { internacionales y reglas } \\
\text { país destino }\end{array}$ & & & \\
\hline $\begin{array}{l}\text { Trámites burocráticos y } \\
\text { trabas legales }\end{array}$ & & & \\
\hline
\end{tabular}

Fuente: elaboración propia. 